\title{
Screening Refusal Associated with Choice of Colorectal Cancer Screening Methods. A Cross-sectional Study Among Swiss Primary Care Physicians
}

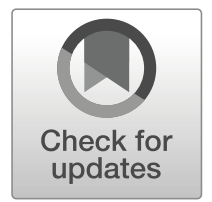

\author{
Yonas Martin, $M D^{1,2}$, Alexander Leonhard Braun, $M D^{7}$, Nikola Biller-Andorno, MD, $P$ hD ${ }^{3}$, \\ Jean-Luc Bulliard, $P h D^{4}$, Jacques Cornuz, MD, MPH ${ }^{5}$, Kevin Selby, MD ${ }^{5}$, and Reto Auer, MD, MAS $S^{1,5}$ \\ 'Institute of Primary Health Care (BIHAM), University of Bern, Bern, Switzerland; 2Department of General Internal Medicine, Inselspital, Bern University \\ Hospital, University of Bern, Bern, Switzerland; ${ }^{3}$ Institute for Biomedical Ethics and History of Medicine (IBME), UZH, Zürich, Switzerland; ${ }^{4}$ Institute of \\ Social and Preventive Medicine (IUMSP), University of Lausanne, Lausanne, Switzerland; ${ }^{5}$ Department of Ambulatory Care and Community \\ Medicine, University of Lausanne, Lausanne, Switzerland.
}

J Gen Intern Med 34(8):1409-11

DOI: $10.1007 / \mathrm{s} 11606-019-05096-2$

(c) Society of General Internal Medicine 2019

\section{INTRODUCTION}

Guidelines recommend primary care physicians (PCPs) offer patients a choice of colorectal cancer (CRC) screening methods, including colonoscopy and fecal occult blood tests (FOBT). ${ }^{1}$ However, in countries like the USA and Switzerland, patients are screened almost exclusively with colonoscopy. ${ }^{2,3}$ When offered both tests, patients appear as likely to choose one as the other; the predominance of colonoscopy may largely be explained by physician preference and local medical culture. ${ }^{4}$ Offering only colonoscopy might explain why screening rates are low.

We sought to determine the proportions of patients who opted for screening with colonoscopy or FOBT and who refused testing among 50-75 year olds eligible for screening at a PCP visit. We described variation in care between PCPs and tried to identify PCP-level factors associated with testing method and refusal.

\section{METHODS}

We conducted a cross-sectional data collection on CRC screening practices at PCP level. We invited 129 PCPs from the Swiss Sentinel Surveillance Network (Sentinella) to fill a structured data collection form for 40 consecutive non-emergent consultations with 50-75-year-old patients. The federal office of public health (FOPH) provided demographic data at PCP level.

$\overline{\text { Prior Presentations An earlier version of the abstract was presented at }}$ the Wennberg International Conference (WIC) in Zürich, Switzerland in April 2018 and at the Swiss Society of General Internal Medicine (SSGIM) meeting in Basel, Switzerland in May 2018. The abstract was presented at the European Society of Medical Decision Making (SMDM) conference in Leiden, Netherlands, in June 2018. This work was presented as oral presentation at the American Society of Medical Decision Making (SMDM) Conference in Montreal in October 2018.

Published online June 12, 2019
PCPs reported demographic data at patient-level, data on previous $\mathrm{CRC}$ tests, contraindications for screening, risk factors for CRC, if CRC screening was discussed, choice of test (colonoscopy, FOBT, other), and refusal for testing. We calculated overall proportions and reported variation between PCPs in the proportion of FOBT vs. colonoscopy they prescribed to patients who chose to be tested. We calculated overall prescription rates of FOBT vs. colonoscopy for each PCP, including both patients who had already undergone screening and patients prescribed screening after the consultation. We dichotomized this covariate by never-prescription of FOBT (no patients previously tested with FOBT or prescribed FOBT after discussion) vs. any FOBT.

We used mixed-effects logistic regression models that allowed us to cluster the data by PCP (with PCPs modeled as a random effect) to explore the association between PCP characteristics and the proportion of patients who refused screening after discussion. We adjusted the models for PCPs' demographics (age, sex) and language region, for patients' demographics (age, sex), and PCPs' prescription patterns.

\section{RESULTS}

Ninety-one PCPs (71\% of invited, mean age 54, 24\% women) collected data on 3637 patients. One hundred eighty-six patients were excluded because they were not aged $50-75$ years old or had already been seen during data collection. The analysis included 3453 patients (mean age 63, 50\% women). PCPs discussed screening with $51 \%(874 / 1727)$ of eligible patients (not up-to-date and no contraindications for testing) (Fig. 1). After excluding patients with risk factors or symptoms suggestive of CRC ( $n=104), 61 \%$ (473/770) opted for screening (FOBT/colonoscopy ratio 0.5 ), 29\% refused, $6 \%$ were undecided, and 3\% were unspecified or missing. Most patients who refused screening said they did so because they did not feel concerned.

Thirty-three PCPs (36\%) had none of their patients previously tested with FOBT or who planned to be tested with FOBT. Patients of PCPs who only offered colonoscopy were 


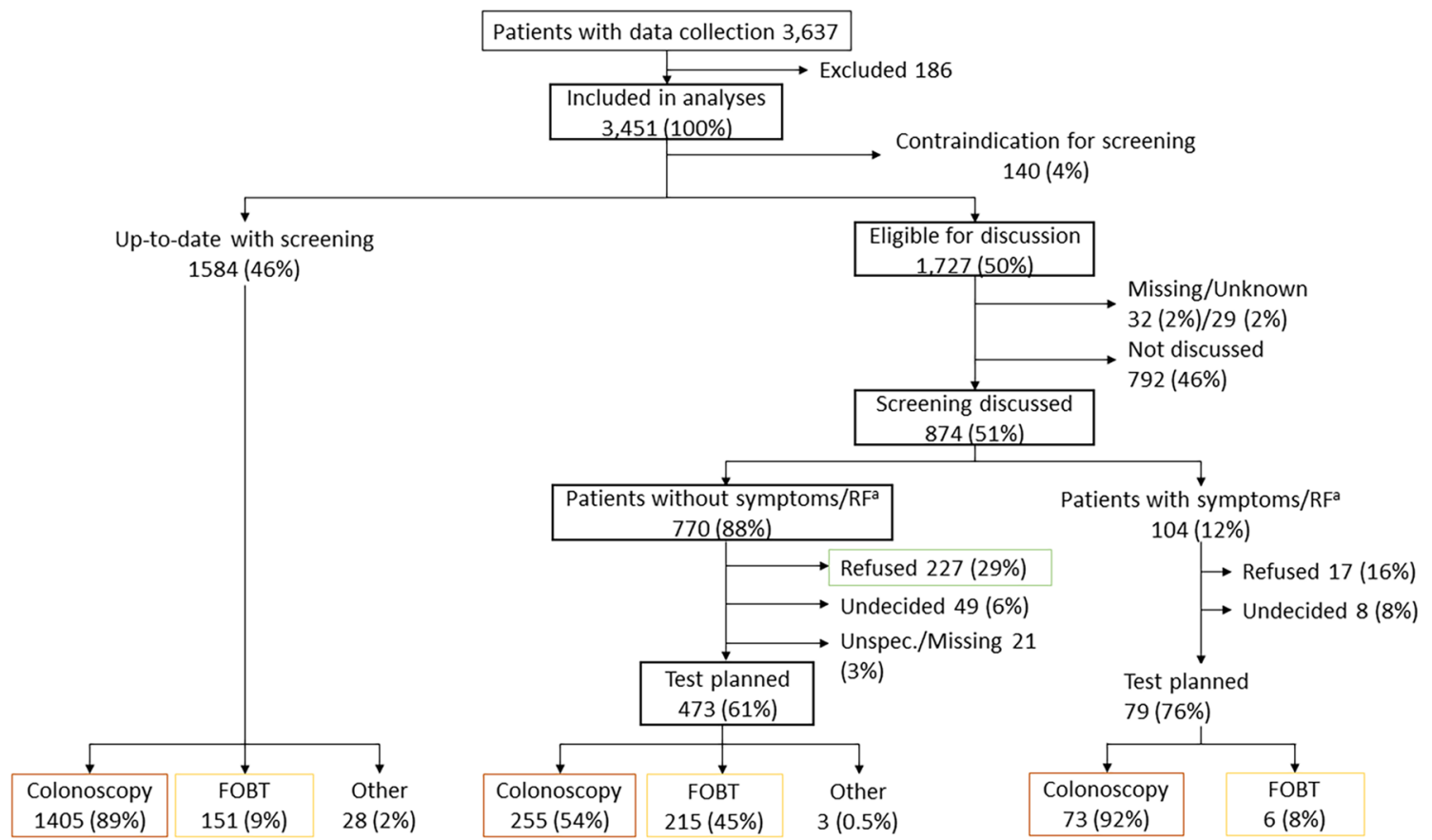

Figure 1 Flowchart of 40 consecutive patients aged 50-75 included by PCPs from the Sentinella network in 2017. PCPs collected data on 40 consecutive patients aged 50-75 from on past screening status, contraindications for screening, if a discussion on CRC screening could take place, RF and symptoms for CRC, and the decision taken (refusal, FOBT, colonoscopy, other). Data collected between April and December 2017. ${ }^{\mathrm{a}} \mathrm{RF}$, risk factor for CRC.

more likely to refuse screening than patients of PCPs who offered both colonoscopy and FOBT (44\% vs. 20\%, respectively, Fig. 2). These results were confirmed in our mixedeffects multivariate model (OR 3.90, 95\% CI 1.90 to 8.00 , $p<0.001)$. No other PCP characteristics were associated with chosen testing methods or refusal rates.

\section{DISCUSSION}

When PCPs discussed CRC screening with their 50-75year-old patients who were not up-to-date with screening, had no contraindication, and no risk factors for CRC, a third of their patients declined to be screened. PCPs who only offered colonoscopy had lower screening rates $(47 \%$

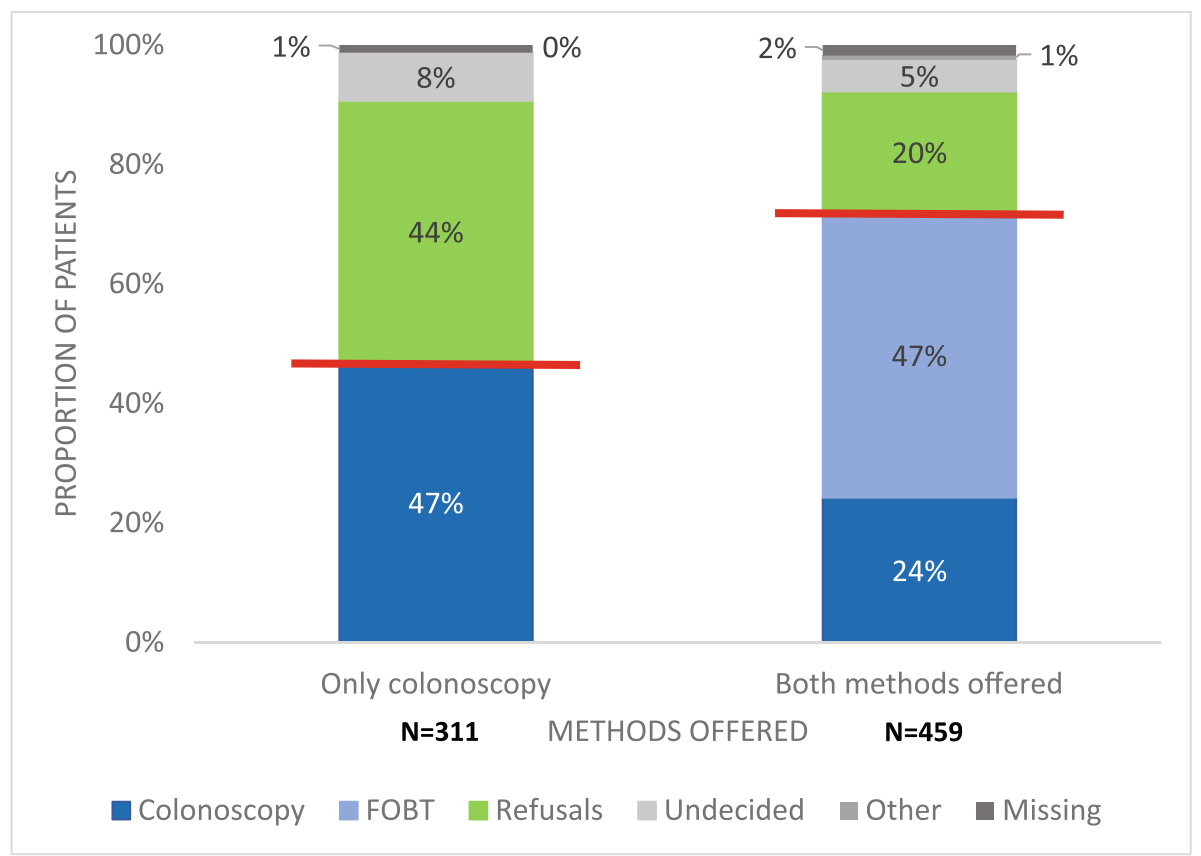

Figure 2 Decision patterns among patients who had a discussion on CRC screening $(N$ patients $=770)$ and included by PCPs who only prescribed colonoscopy $(N=33)$ vs. PCPs who prescribed both colonoscopy and FOBT $(N=58)$, in the Sentinella Network in 2017. Patients with risk factors or symptoms suggestive for CRC $(n=104)$ (see Fig. 1) excluded of this analysis. 
vs. 71\%) and higher refusal rates (44\% vs. 20\%) than PCPs who offered both colonoscopy and FOBT. These results are in line with a randomized controlled trial showing lower uptake rates of CRC screening tests among patients who are offered only colonoscopy vs. among the ones who are offered both FOBT and colonoscopy. ${ }^{5}$ We were inherently limited in considering additional patient-level sociodemographic factors by the simplicity and anonymity of our data collection.

Encouraging PCPs to offer both methods could reduce the number of physicians who only prescribe one screening modality, reduce variation between practices, and allow more patients to choose the test that matches their preferences and values. ${ }^{4,6}$ This could reduce the number of refusals, raise CRC screening rates, and ultimately lower the burden of CRC.

Acknowledgements: We thank the primary care physicians from the Sentinella network and the Sentinella administration as well as the Section Notification Systems at the Federal Office of Public Health for their help collecting this data. We thank Kali Tal for the thorough editing of this work. We thank Emanuele Prati for his help in the data collection. We thank Charles Dvorák for the great help in the conduction of the study among Sentinella PCPs. We thank Cyril Ducros for his comments to this work.

Corresponding Author: Yonas Martin, MD; Institute of Primary Health Care (BIHAM), University of Bern, Bern, Switzerland (e-mail: yonas.martin@biham.unibe.ch).

Funders This work was supported by the funds from the Swiss National Scientific Foundations National Research Plan 74 NFP74. 407440_167519. The funders had no role in the design or conduct of the study; in the collection, management, analysis, or interpretation of data; nor in the preparation, review, or approval of the manuscript.

\section{Compliance with Ethical Standards:}

Conflict of Interest: None of the authors has a conflict of interest related to this manuscript.
Open Access This article is distributed under the terms of the Creative Commons Attribution 4.0 International License (http:// creativecommons.org/licenses/by/4.0/), which permits unrestricted use, distribution, and reproduction in any medium, provided you give appropriate credit to the original author(s) and the source, provide a link to the Creative Commons license, and indicate if changes were made.

\section{REFERENCES}

1. Bibbins-Domingo K, Grossman DC, Curry SJ, Davidson KW, Epling JW, Jr., Garcia FAR, et al. Screening for Colorectal Cancer: US Preventive Services Task Force Recommendation Statement. JAMA. 2016;315(23):2564-75. doi:https://doi.org/10.1001/jama.2016.5989

2. Fedewa SA, Cullati S, Bouchardy C, Welle I, Burton-Jeangros C, Manor O, et al. Colorectal Cancer Screening in Switzerland: Cross-Sectional Trends (2007-2012) in Socioeconomic Disparities. PLoS One 2015;10(7):e0131205. doi:https://doi.org/10.1371/journal.pone. 0131205

3. McQueen A, Bartholomew LK, Greisinger AJ, Medina GG, Hawley ST, Haidet $\mathbf{P}$, et al. Behind Closed Doors: Physician-Patient Discussions About Colorectal Cancer Screening. J Gen Intern Med 2009;24(11):122835. doi:https://doi.org/10.1007/s11606-009-1108-4

4. Selby K, Cornuz J, Gachoud D, Bulliard JL, Nichita C, Dorta G, et al. Training primary care physicians to offer their patients faecal occult blood testing and colonoscopy for colorectal cancer screening on an equal basis: a pilot intervention with before-after and parallel group surveys. BMJ Open 2016;6(5):e011086. doi:https://doi.org/10.1136/bmjopen-2016-011086

5. Inadomi JM, Vijan S, Janz NK, Fagerlin A, Thomas JP, Lin YV, et al. Adherence to colorectal cancer screening: a randomized clinical trial of competing strategies. Arch Intern Med 2012;172(7):575-82. doi:https:// doi.org/10.1001/archinternmed.2012.332

6. Mulley AG, Trimble C, Elwyn G. Stop the silent misdiagnosis: patients' preferences matter. Bmj. 2012;345:e6572.

Publisher's Note Springer Nature remains neutral with regard to jurisdictional claims in published maps and institutional affiliations. 